\title{
CT Hypoperfusion Complex: Emergency CT Results During One Year
}

İhsan Yüce

Department of Radiology, Atatürk University School of Medicine, Erzurum, Turkey

\begin{abstract}
Aim: Computed tomography (CT) hypoperfusion complex is observed in the imaging findings of intra-abdominal organs and vascular structures during shock and hypoperfusion. The aim is to examine the frequency of CT hypoperfusion complex and etiologic factors by retrospectively analyzing the CT performed on patients admitted to emergency departments.
\end{abstract}

Materials and Methods: In our study, 930 abdomen and thorax tomographies were obtained from the patients admitted to our emergency department during a 1-year period. All CT scans were performed by using a 640-slice tomography device. The CT criteria for hypoperfusion complex included the small-caliber aorta and inferior vena cava (IVC), hyperdense bowel wall, surrenal enhancement, hypoperfused liver, spleen, and hyperdense kidney.

Results: Our study revealed the presence of CT hypoperfusion complex in at least 1 of 15 patients (0.16\%). We detected low-calibrated abdominal aorta together with hyperdense adrenal glands in all the CT hypoperfusion complex cases ( 15 cases, $0.16 \%$ ). We found the presence of intra-abdominal free fluid in 13 cases $(0.13 \%)$, halo sign in IVC in 8 cases $(0.08 \%)$, hypoperfusion view of the spleen and liver in 5 cases $(0.05 \%)$, pericholecystic fluid in 3 cases $(0.03 \%)$, and sign of renal hyperperfusion (white kidney sign) in 3 cases $(0.03 \%)$.

Conclusion: Our study showed that low-calibrated abdominal aorta and hyperdense adrenal glands were present in each patient showing CT hypoperfusion complex, and the assessment of these two structures is very important before the setting-in of irreversible shock.

Keywords: Computed tomography, hypoperfusion, shock

\section{Introduction}

Computed tomography (CT) hypoperfusion complex is seen particularly in intra-abdominal organs and vascular structures as a result of shock and hypoperfusion (1-10). It was first defined in pediatric patients with multiple traumas by Taylor et al. (1). Besides trauma, it can also be observed during sepsis, diabetic ketoacidosis, and myocardial infarction (MI) (2). Since cardiac output and arterial pressure can be sustained in the compensatory phase of shock, blood pressure and pulse parameters alone may be insufficient to explain the patient's state. The evaluation of imaging findings is of great importance in such circumstances. In this article, we aimed to examine the frequency of CT hypoperfusion complex and etiologic factors by retrospectively analyzing the abdomen and thorax tomographies performed on patients admitted to emergency departments for various reasons between 2014 and 2015.

\section{Materials and Methods}

\section{Patients' population}

In our study, we retrospectively evaluated 930 abdomen and thorax tomographies obtained from patients admitted to our emergency department for various reasons between October 2014 and October 2015.

\section{CT imaging}

All these patients underwent abdomen and/or thorax tomography by means of 640-slice CT (Aquilion ONE ViSION, Toshiba, Japan). Approximately $100 \mathrm{cc}$ of iodinated contrast media $(3.5 \mathrm{cc} / \mathrm{s}$ ) was injected into the patients from the left antecubital region by means of auto-injectors. Portal-phase scan was performed for patients undergoing abdominal tomography, and arterial-phase scan was performed for patients undergoing abdominal thorax tomography. 


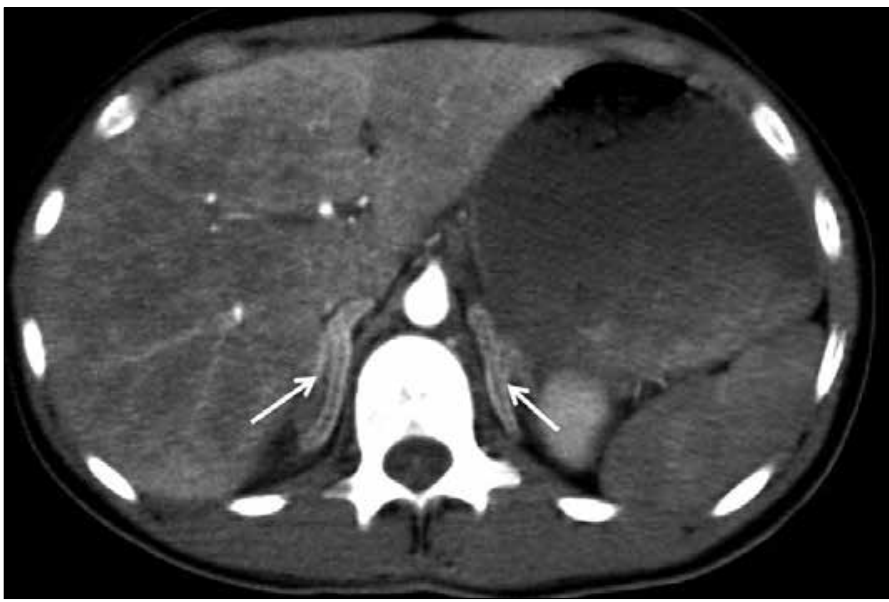

Figure 1. Hyperdense adrenal glands are seen in axial CT image (arrows)

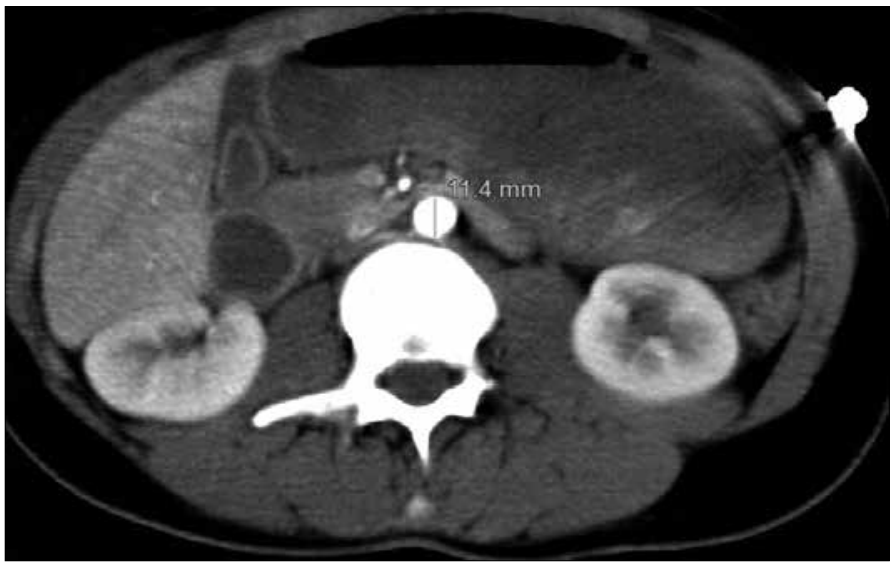

Figure 2. Low-calibrated abdominal aorta is seen in axial CT image. It must be less than $13 \mathrm{~mm}$ in measurements performed $2 \mathrm{~cm}$ below and above the renal arteries (3)

\section{CT evaluation}

For the diagnosis of low-calibrated abdominal aorta, the diameter measurements of the abdominal aorta of the renal arteries $2 \mathrm{~cm}$ below and above were considered to be the diagnostic criteria (3). For low-calibrated IVC, the average was calculated by measuring 3 different regions of the intrahepatic vena cava: measurements less than 9 $\mathrm{mm}$ were considered to be the diagnostic criteria (3). For hyperdense adrenal glands, the state of being equal or hyperdense when compared to peripheral vascular structures (e.g., IVC) and holding more contrast than the spleen and liver were considered to be the diagnostic criteria (3). For the diagnosis of shock kidney, extended and contrast kidneys were considered to be the diagnostic criteria. For hypoperfused spleen and liver, an increase in the heterogeneous area (perfusion defects) when compared to normal parenchyma was considered to be the diagnostic criterion. All CT images were evaluated by a radiologist with 8 years of experience in emergency radiology (IY).

\section{Results}

In our study, 930 abdominal and thorax tomographies of pediatric and adult patients were evaluated. In these, 544 patients were male (58\%) and 386 patients were female (41\%). The mean age of the patients was $32 \pm 5.3$ years. We found the presence of CT hypoperfu-

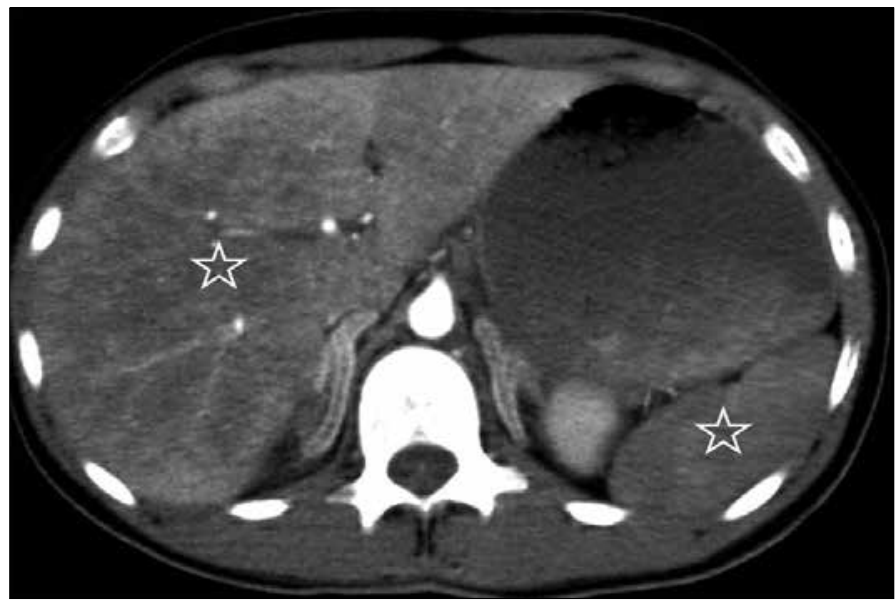

Figure 3. Perfusion defects in the liver and spleen are seen in axial CT image (stars)

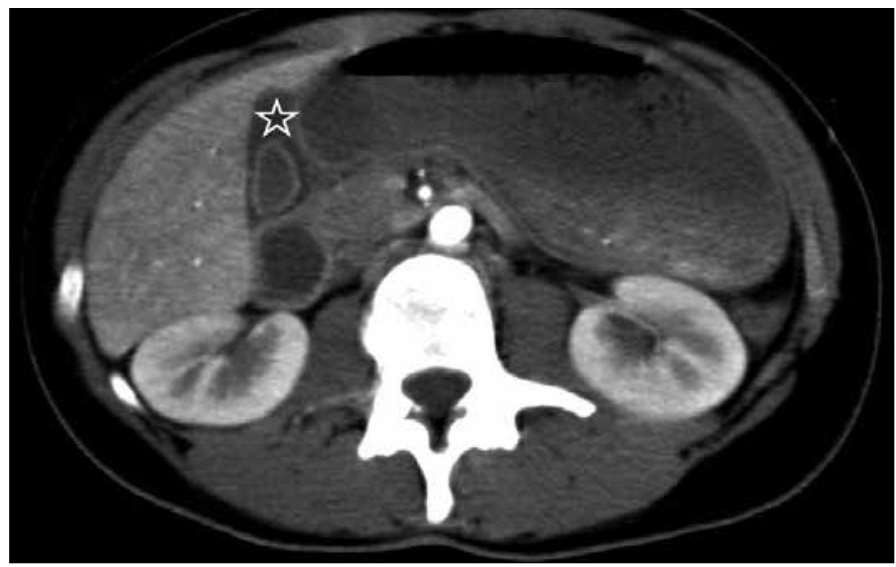

Figure 4. Pericholecystic fluid is seen in axial CT image (star)

sion complex in at least 1 of 15 patients (0.16\%). All of these patients' vital signs were stable (they might have been in the compensatory phase of shock). Further, out of the 15 patients, 12 patients suffered from multiple traumas $(0.12 \%), 2$ patients were in the post-operative stage $(0.02 \%)$, and 1 patient $(0.01 \%)$ suffered from sepsis. We observed low-calibrated abdominal aorta together with hyperdense adrenal glands (15 cases, $0.16 \%$ ) (Figure 1,2) in all the patients with CT hypoperfusion complex. We detected intra-abdominal free fluid in 13 cases $(0.13 \%)$, halo sign in the IVC in 8 cases $(0.08 \%)$, hypoperfused spleen and liver in 5 cases (0.05\%) (Figure 3), pericholecystic fluid in 3 cases $(0.03 \%)$ (Figure 4$)$, and sign of renal hyperperfusion (white kidney sign) in 3 cases (0.03\%) (Figure 5). Apart from this, low-calibrated thoracic aorta and collapsed heart appearance observed in thorax-related results were present in 5 cases $(0.05 \%)$. On the other hand, 1 patient died because of septic shock. Other patients recovered fully after a 3-month follow up.

\section{Discussion}

Computed tomography hypoperfusion complex is a set of tomographic results that was defined by Taylor et al. (1) for the first time in 1987. Basically, it is the definition of hypoperfusion results seen in abdominal and, sometimes, thoracic structures as a result of circulation 


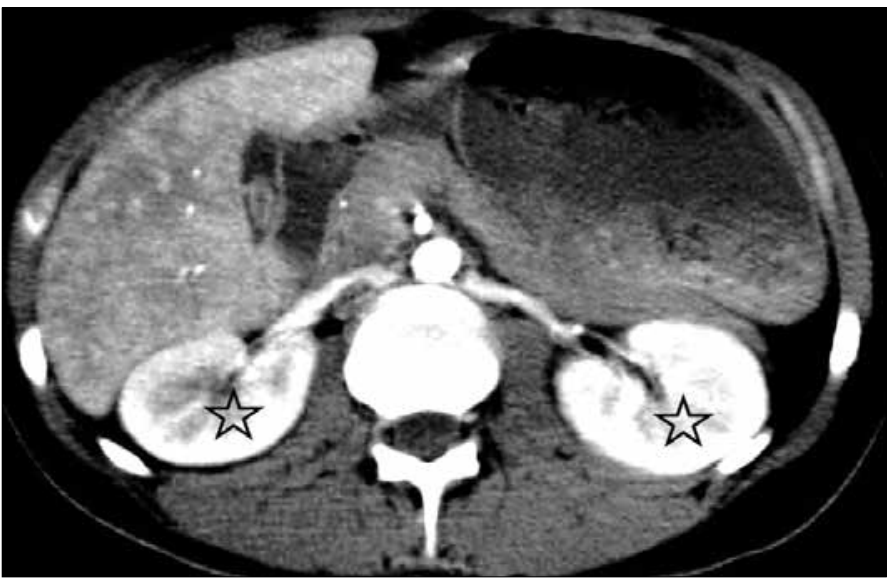

Figure 5. White kidney sign is seen in axial CT image (stars)

guidance from splanchnic circulation to vital organs due to the activation of the sympathetic system post-shock (hypovolemia). At the same time, CT hypoperfusion complex was found to be associated with poor prognosis (3). However, it is known that emergency physicians and even radiologists have difficulties in defining these findings.

There are three basic stages of shock: compensatory, decompensatory, and irreversible. Arterial pressure, in particular, can be maintained in the compensatory phase, and monitoring of only the vital signs is not enough to explain the patient's state. In abdominal tomography performed at this stage, the presence of CT hypoperfusion complex in the patient indicates that the patient is in shock and there must be an appropriate treatment for this exigency. At the same time, understanding the signs of CT hypoperfusion prevents confusion with pathologies that can enter the differential diagnosis, including ileus, gallbladder perforation, mesenteric ischemia, portal venous thrombosis, and DIC.

The signs of CT hypoperfusion complex can be divided into two: vascular and non-vascular. Low-calibrated abdominal aorta, IVC, and mesenteric arteries can be categorized as vascular signs. Dilated and edematous bowel loops, hyperdense adrenal glands, shock kidney, shock pancreas, free fluids in the abdomen, splenic and hepatic perfusion deficits, and pericholecystic fluid can be categorized as non-vascular signs. Apart from these findings about the abdomen, low-calibrated thoracic aorta and loss of volume in cardiac chambers (in extreme cases) can also be considered.

Certainly, some measurements are also needed to be made for the evaluation of these signs. For instance, the following can be considered to be diagnostic criteria: $(A)$ in the diagnosis of low-calibrated abdominal aorta, the abdominal aorta diameter of the renal arteries $2 \mathrm{~cm}$ below and above is less than $13 \mathrm{~mm}$; (B) with regard to low-calibrated IVC, the IVC diameter is less than $9 \mathrm{~mm}$ in the measurements made from 3 different regions of the intrahepatic vena cava; $(C)$ in the case of hyperdense adrenal glands, the state of being equal or hyperdense when compared to peripheral vascular structures (e.g., IVC) and holding more contrast than the spleen and liver; (D) in the case of shock kidney diagnosis, extended and significantly more hyperdense kidneys; and (E) in the case of hypoperfused spleen and liver, increase in the heterogeneous area (perfusion defects) when compared to normal parenchyma (3).

In our study, we emphasize the observation of hyperdense adrenal glands and low-calibrated abdominal aorta signs in all patients whose imaging reveals CT hypoperfusion complex (sensitivity: 100\%; specificity: $100 \%)$. Therefore, the observation of hyperdense adrenal glands together with low-calibrated abdominal aorta in portal-phase abdominal tomography (early portal phase is used to observe the arteries) obtained from patients suspected to be in the compensatory phase of shock can confirm that the patient is in shock. Emergency physicians should be alert during this phase of shock. It has been assumed that the above is caused by hypovolemia, and the appropriate treatment is supportive therapy rather than surgical intervention.

The incidence of complex CT hypoperfusion has been reported differently in several studies. However, CT hypoperfusion complex occurs at a higher rate in post-traumatic patients. In our study, 12 out of the 15 cases with CT findings consisted of post-traumatic cases. In a study conducted on post-traumatic cases by Ryan et al. (3), CT hypoperfusion complex was seen in 27 out of 498 patients (5.5\%). Again, CT findings in a pediatric group with blunt abdominal trauma were evaluated by Taylor et al. (1), and they reported the incidence as $0.01 \%$. In our study, this incidence is $0.16 \%$.

\section{Conclusion}

Computed tomography hypoperfusion complex is a set of imaging signs that is even difficult for radiologists to identify. However, if identified, it can lead to appropriate treatment along with diagnosis even in patients in the compensatory phase of shock. In addition, the observation of hyperdense adrenal glands together with low-calibrated abdominal aorta in abdominal CT scans can confirm that the patient is in shock, and we should check at least these two structures in patients suspected to be in shock.

Ethics Committee Approval: Authors declared that the research was conducted according to the principles of the World Medical Association Declaration of Helsinki "Ethical Principles for Medical Research Involving Human Subjects", (amended in October 2013).

Informed Consent: Informed constent was not obtained because our study was a retrospective study.

Peer-review: Externally peer-reviewed.

Conflict of Interest: No conflict of interest was declared by the authors.

Financial Disclosure: The authors declared that this study has received no financial support.

\section{References}

1. Taylor GA, Fallat ME, Eichelberger MR. Hypovolemic shock in children: abdominal CT manifestations. Radiology 1987; 164: 479-81. [CrossRef]

2. Mirvis SE, Shanmuganathan K, Erb R. Diffuse small-bowel ischemia in hypotensive adults after blunt trauma (shock bowel): CT findings and clinical significance. AJR 1994; 163: 1375-9. [CrossRef]

3. Ryan MF, Hamilton PA, Sarrazin J, Chu P, Benjaminov O, Lam K. The halo sign and peripancreatic fluid: useful CT signs of hypovolaemic shock complex in adults. Clin Radiol 2005; 60: 599-60. [CrossRef]

4. Hara H, Babyn PS, Bourgeois D. Significance of bowel wall enhancement on CT following blunt abdominal trauma in childhood. J Comput Assist Tomogr 1992; 16: 94-8. [CrossRef]

5. Tarrant AM, Ryan MF, Hamilton PA, Benjaminov O. A pictorial review of hypovolaemic shock in adults. Br J Radiol 2008; 81: 252-7. [CrossRef]

6. Lubner M, Demertzis J, Lee JY, Appleton CM, Bhalla S, Menias CO. CT evaluation of shock viscera: a pictorial review. Emerg Radiol 2008; 15: 1-11. [CrossRef]

7. Landreneau RJ, Fry WJ. The right colon as a target organ of nonocclusive mesenteric ischemia: case report and review of the literature. Arch Surg 1990; 125: 591-4. [CrossRef]

8. Shamji FM, Todd TR. Hypovolemic shock. Crit Care Clin 1985; 1: 609-29.

9. Bulkley GB, Kvietys PR, Parks DA, Perry MA, Granger DN. Relationship of blood flow and oxygen consumption to ischemic injury in the canine small intestine. Gastroenterology 1985; 89: 852-7. [CrossRef]

10. Brochert A, Rafoth JB. Shock thyroid: a new manifestation of the hypovolemic shock complex in trauma patients. J Comput Assist Tomogr 2006; 30: 310-2. [CrossRef] 